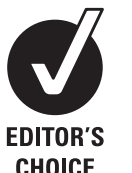

CHOICE

Correspondence to

Dr Catherine J Quarini;

catherinequarini@doctors.org.uk

CQ is a CT1 doctor working in the Oxford Deanery. The situations described are not based on any specific individuals or hospital wards, but are instead a representation of the general experiences of many junior doctors at a variety of different hospitals around the country. Details relating to patients and doctors have been changed to ensure anonymity as per the BMJ guidelines.

Received 17 May 2009 Accepted 3 May 2010 Published Online First 20 August 2010

\title{
A day in the life of a junior doctor: everyday ethical encounters
}

\author{
Catherine J Ouarini
}

\section{ABSTRACT}

This paper presents a hypothetical 'day in the life' of junior doctors working on a busy hospital ward. It illustrates the fact that, although the everyday ethical encounters faced by doctors are generally not as dramatic as some of the ethical issues discussed at medical school, the underlying principles, such as consent, confidentiality and resource allocation, are highly relevant to daily practice. After presenting some of the ethical challenges faced by junior doctors, from patients confused by poor explanations to inadequate consent procedures, the paper ends with suggestions on how to improve the situation.

\section{INTRODUCTION}

The primary aim of this paper is to raise awareness of the ethical issues faced by junior doctors by presenting a typical 'day in the life' of junior doctors working on a hospital ward. The situations described are not based on any specific individuals or hospital wards, but are instead a representation of the general experiences of many junior doctors at a variety of different hospitals. What emerges is the importance of paying attention to the everyday issues of medical ethics and how poor ethical standards are closely linked to the delivery of routine care. The paper is broadly divided into two parts. The first describes a junior doctor's typical day. The second addresses the practical implications of this account.

\section{THE START OF THE DAY}

It is the start of another busy day in the lives of a group of hospital-based junior doctors. Everyone is rushing in, hoping to arrive before their consultant. The morning begins with a meeting of all the doctors working on that hospital ward. The purpose of the meeting is to share any new information from the previous day and ensure that everyone is updated on new patients before the morning ward round. Today's meeting, as usual, involves an informal get-together at the 'nurses station'-a work bench with computers usually situated near the entrance to the ward and no more than a couple of metres away from patients' beds. There is little privacy for patients. The first thing that the junior doctors do is add the details of the new patients to their list, including their presenting complaints and basic medical history. Next, any updates on patients' conditions are discussed, and problems experienced with 'difficult' patients and relatives are shared. Many patients, still in their beds, are within earshot of the conversation and others are walking past on their way to the bathroom.
This meeting is the first place where confidentiality is breached. Not only are patients likely to overhear private information, but the computer screen on which their personal information is displayed often does not get closed down in the rush to get to the ward round, remaining on the desktop for passing pharmacists, physiotherapists, medical students and anyone else to view.

\section{THE WARD ROUND}

This first breach of confidentiality might be deemed a relatively minor matter, when viewed in light of the main part of the morning's activities, the ward round. This involves all the doctors on the team, usually a consultant, registrar, foundation doctors and often a medical student, seeing each patient on the ward, checking their progress, and organising management plans such as further investigations, medication or discharge. There are generally about six beds in each room, the only privacy being a curtain that can be pulled around the bed. This set-up presents a huge problem with maintaining confidentiality-the doctors who do remember to pull the curtains around each bed still do not manage to sound-proof the curtain before talking to the patient, so the whole room hears the symptoms, likely diagnosis, and answers to any questions the doctors may ask about bowel habits, alcohol intake, or sexual history of their ward neighbours. Since the patient knows the answers are shared with the rest of the room, the honesty and completeness of the answers may be open to question.

The nature of the doctor-patient relationship is another important ethical issue. During each 10 min consultation on the ward round, the consultant will dictate a list of investigations to be carried out and drugs to be given. The drugs will be prescribed by one of the junior doctors on the 'drug chart', while another writes an entry in the medical notes detailing the plan, and another makes a list of investigations to be organised. All this frantic writing is performed during the brief consultation. During most of these ward-round visits, nothing is discussed with the patient in any detail. Today, as usual, the consultant directs most of the questions about the patient's condition to the junior doctors, not to the patient. Any questions asked of the patient are usually brief, closed questions, with minimal eye contact and body language making it clear that the doctors are in a hurry and have 30 people to see before lunchtime.

Plans for further tests or medication changes are stated as undisputed facts, not something to be discussed, and sometimes these changes are suggested as the medical team is walking away 
from the bed, with the patient not being aware of them at all. Once a drug is prescribed or a test ordered, nurses dispense the drug and porters will collect the person for a scan, often without the patient being aware of what drug they are being given, or what type of scan they are having or why. The rare, daring patient who does ask questions on this ward round is usually given brief answers. The man in bed 1 fits into this category, and, as the doctors begin to walk away, still discussing his hypertension and its possible causes, he asks for an explanation of what they are talking about and what they have planned. He is told by the consultant that he is being given 'a blood pressure pill'. That is the extent of the information volunteered-possible side effects and alternatives are not discussed.

This presents a dilemma for the junior doctors who are accustomed to a more deliberative style of doctor-patient relationship. They are concerned about the lack of patient autonomy. They can tell that the man is unhappy with the lack of information and that he has not given valid consent for the investigations suggested. One of the junior doctors makes a note on her list to return later in the afternoon and ask if he has any questions or concerns. This, however, is not going to happen for several hours, as there are still 29 more people to see.

The doctors proceed to bed 2. Here, codes of professional ethics and General Medical Council (GMC) guidelines ${ }^{1}$ are further tested: a medical student who needs to be signed off as having done a normal cardiovascular examination is introduced by the consultant to the patient as 'a colleague who needs to examine your heart'. In such cases, some medical students interrupt with 'I'm a medical student and I'm just listening to your heart as practice, not because we think there is anything wrong', but that does not happen today. This lady has not come in with any cardiovascular complaints, nor has she ever suffered from any heart problems, so she seems worried that someone, whom she assumes is a doctor, has been asked to come with a group of other doctors to examine her heart. She asks if her blood test results showed any abnormalities, as this is the only way she thinks anyone could have picked up on a heart condition. "Don't worry about those" she is told.

Several patients later and the ward round has reached bed 8. An elderly man has been admitted with several minor leg injuries following a fall. He also has obvious rheumatoid arthritis in his hands, with pronounced signs of the disease. The registrar is interested as he is later giving a rheumatology lecture, and a photo would look good in his presentation. He does not share these thoughts with the patient, but asks him 'May I take a photo of your hands?' When the puzzled man, doubtless wondering what his deformed hands have to do with his leg injuries, nods, the registrar takes a photo on his phone to use in the presentation. Conversations about consent for presentation of these photos, and who they may be seen by, or emailed to later, are omitted, as are any thoughts of the principles of research and publication ethics or GMC guidelines requiring patients to give permission for photos to be taken. ${ }^{2}$ Meanwhile, everyone rushes on.

\section{COMMON DISTORTIONS}

Several hours later, the ward round is finished. All the patients have been seen, and each of the junior doctors has a list of tasks: to organise follow-up, scans, treatments and blood tests. Once the consultant has left, the other doctors go through the list of patients to decide who will do what. They agree that some concerns emerged during the ward round which now need to be addressed, so they add 'reassure lady in bed 2 that her heart is $\mathrm{OK}$ ' and 'explain medications to man in bed 1' to their list, in addition to the tasks given to them by the consultant.
During a discussion about investigations, the doctor who has volunteered to go to the radiology department to book all the scans for the ward notices that the ultrasound scan that the consultant requested for the patient in bed 17 (to investigate his abnormal blood test results) should actually be for the person in bed 18; the consultant seems to have confused the two. However, the doctors agree that, although it is a waste of resources, they should still request the unnecessary ultrasound scan for the person in bed 17, in addition to the correct one for the person in bed 18. It is preferable to do this, rather than have the consultant in a bad mood during the next ward round and claiming that they did not listen to his instructions. As none of the patients heard which scans they were supposed to be having or whether they were having scans at all, this is an easy addition to make.

The junior doctor returns from the radiology department pleased that, for once, the radiologist agreed to do all the scans the consultant wanted, including a couple of CT scans and an MRI scan. This feat required a slight exaggeration of the patients' symptoms to elevate them to 'red flag' status. These are symptoms that are known to be possible signs of serious illness, so the radiologist finds it difficult to refuse to carry out the scans, as it would be hard to defend this course of action at a later date if it became necessary. The doctor is aware of his manipulation and the questionable use of resources within the health service. He also knows that the symptoms his patients were experiencing do not justify these scans, but he reconciles his actions to himself by deciding that all his colleagues do the same thing and therefore his patients will be at the back of the queue if he does not do the same.

\section{UNINFORMED CONSENT}

Taking consent for procedures is one of the least popular tasks. Most junior doctors are aware of the legal and ethical recommendations that the person carrying out the procedure, such as the surgeon, should be the one to take consent, and in their absence it should be someone who can perform that procedure themselves. However, today, as on most days, an irate theatre nurse phones the ward demanding to speak to a doctor. She says that the anaesthetist and the surgeon need the patient in the operating theatre in $30 \mathrm{~min}$ and cannot do anything without a signed consent form. The junior doctor knows that this is a planned operation, so the consent form could have been completed at an outpatient clinic or on the ward. After attempting to contact the consultant and registrar and hearing answer-phone messages both times, he decides that unless he fills in the consent form, the patient will miss their operation entirely. He quickly searches for the condition and the proposed operation on the internet and tries to ascertain the most common and most serious risks of the procedure.

The junior doctor goes through the form carefully with the patient, explaining the benefits and possible complications of the surgery. He hopes that his lack of first-hand experience of the procedure may be offset by giving the patient time to read the form and ask questions, things that are rarely encouraged if consent forms are completed on a busy ward round.

Unfortunately, the elderly patient is quite deaf, and asks the doctor to repeat some of the information more slowly and loudly, which he does. She understands the treatment that is being proposed and the principal benefits and risks of the surgery, including the consequences of not having the procedure, and the doctor is satisfied that she has the capacity to make a decision. The patient says she did not realise what the surgery involved, nor did she appreciate the recovery time, and she begins to ask a question when her family interrupt her. They say 
to the doctor 'Do whatever you think is best, she won't understand anyway' and instruct their relative to 'Just sign the form. The doctors are busy'. The doctor knows that consent should be voluntary and free from coercion and that it would be preferable to speak to the patient on her own, without the intrusion of her family.

However, as they had responded to his first request to be left alone with the patient with "We're not going anywhere-we've had time off work to be here and we've been kept waiting", he decides that the limited time available would be put to better use going through the consent form with the patient, rather than fending off angry relatives.

The porters have arrived to collect the patient, who quickly signs the form, and tells the doctor and nurse that she will go ahead as she would 'not like to waste anyone's time', or 'upset the GP' who referred her, or make her 'family angry'. When the doctor follows the bed down the ward still explaining to her that it is her choice and no-one will be angry, she says she understands and has made her mind up.

\section{THE WHOLE TRUTH}

Walking back through the ward, an elderly patient's daughter is waiting to catch the passing junior doctor with a barrage of questions. She wants to speak to the doctor in a private room, away from her father, the patient, as she says she does not want to frighten him with the details of his recently diagnosed illness. It emerges that the diagnosis of lung cancer was explained to the daughter by a doctor on a previous ward. Her father was not included in the discussion, nor has his daughter shared the information with him. He is unaware of his diagnosis. As the patient has only just been transferred to the ward, the doctor has never met him before. The doctor fetches the medical records and tries to find out who spoke to the daughter, what was said, and why the patient was not present. On the day the daughter said she was told the news, there is nothing documented in the notes at all. However, the biopsy results were reported on the computer the day before this, so the sequence and timing of events makes sense. The daughter is angry because she says the doctor on Ward 1 said her father had approximately 6 months to live and radiotherapy would start soon, while a doctor she saw on Ward 2, where he was transferred because of lack of beds, told her a week later that he had less than a month to live, that no treatment would be given, and the palliative care team had been informed and would visit him.

The palliative care team have not seen the patient and nothing is recorded in the notes about this discussion-in fact, nothing was written at all during his stay on Ward 2. After an hour spent trying to phone the possible people concerned, it emerges that no referral has been made to either radiotherapy or the palliative care team. This discussion brings up several ethical issues for the doctor. First, he knows his duty of care is to the patient above the family, and at present the patient has not been informed of his diagnosis or possible management options, and has been unable to give any opinion about the treatment plan. The doctor can see no reason why he would have been unable to understand an explanation of his illness, and there is no documentation stating that the patient has asked not to be told. He decides that the patient must be informed of the biopsy results and allowed to make autonomous choices about his ongoing care.

He explains to the patient that he has some results to discuss with him and this would best be done in a private room on the neighbouring ward. The man wants his daughter present too and the doctor breaks the bad news, after which the patient says that he suspected this diagnosis anyway. The doctor is relieved that the family are now discussing options together. The second ethical issue is what should be done about the communication breakdown on previous wards, and discovering why the daughter was informed of the diagnosis before the patient. However, he decides that, in addition to not knowing how to resolve these problems, his concerns will not change anything anyway and, worse still, one of his colleagues may get blamed for something. Best to forget about it.

This difficult consultation is followed by a much simpler one with the woman whose heart was examined by the medical student during the ward round. She is still anxious about her heart and has asked to speak to a doctor. She is relieved that there is nothing wrong, and says it would have saved her a lot of stress if she had known the reason for the examination from the start. She would willingly have consented to it.

\section{ENOUGH INFORMATION?}

There have been quite a few delays today, and some discussions and procedures have taken more time than expected. It is 17:00 by the time a patient who earlier asked to discuss a new problem with a doctor is seen. She is angry that she has had to wait for several hours. All the junior doctor knows about this patient is that she was admitted following an episode of unexplained bleeding and that she has just received the first of three recommended blood transfusions, before investigations to establish the cause the following day. She says that she feels the hospital have been too slow to deal with her at every stage, she has been ignored by staff and no-one has explained properly what is happening. As a result of all this, she says she does not want to discuss anything with the doctor but is self-discharging at once. The doctor says he is concerned about this decision as her problem is continuing and still under investigation. $\mathrm{He}$ would not advise discharge. She will not hear anything more from him, nor listen to a second opinion or discuss the decision with anyone else. The doctor does not feel that she understands the possible serious consequences of her condition and is keen to get a specialist to speak to her. By the time he has tried to contact all the relevant people, and received no replies (apart from one who told him to advise her "to just do what the doctor says"), she has left the ward. He is unsure if he took the appropriate course of action, and wishes that he could have got her to sign a self-discharge form, but they had run out of these forms on the ward and it is too late anyway. He makes an entry in the medical notes detailing what has happened and moves on to the next task on his list.

One of the final tasks of the day is looking up the results of the day's blood tests and filling in forms requesting blood tests for patients for the following morning. This happens on most hospital wards, and is almost always at the end of the day when the junior doctors have already stayed well past their finishing time and are eager to get the final jobs completed quickly. So the blood test request forms are filled in at speed, often with about 20 or so to be done. The forms have a space for the patient's name and hospital number and then many boxes that can be ticked to request certain tests, such as thyroid function tests, liver function tests, blood group, and so on.

In the case of an unknown cause for the presenting symptoms, many different boxes are ticked to cover more possibilities for analysis. Some of the tests requested are for communicable diseases, such as hepatitis B, and therefore present potential ethical dilemmas in terms of the implications of a positive result, and the decision of the patient and the doctor about who else to inform of such a result. Today, the consultant told the junior doctors to get 'full liver screens, including hepatitis' for several 
patients. The relevant boxes are ticked, and the forms left out for the phlebotomist to collect the following morning.

The only consent gained from patients before these blood tests is verbal consent, taken by the phlebotomist, for the procedure of the blood test, not consent for the specific tests being carried out on the blood. This occasionally leads to an ethical dilemma for the junior doctors when they have to inform a patient that they are, for example, hepatitis B positive, and that they should inform anyone else who may be at risk. Patients are sometimes angry that they have been tested for something they did not know about and doctors are left with the dilemma as to whether to disclose this information to the partners of patients.

\section{THE END OF THE DAY}

The day is finally over. As far as a day in the life of a junior doctor goes, it has been quite routine, with no major surprises or unusual events. It would seem that none of the big questions debated in medical ethics were particularly relevant to this average day on a general hospital ward. There were no dilemmas about end-of-life care, abortion, genetic testing, cloning, IVF, or allocation of scarce resources such as organs or intensive care beds. However, during their average, uneventful day, the junior doctors have been in situations where they have been exposed to breaches of confidentiality, seen medical decisions taken without patient consent, seen patients misinformed (or not informed at all) as to the purpose of their examination or photograph of their signs of illness, and watched several consultations in which the patient was ignored to the extent that they may as well have been completely absent.

The junior doctors have had to make ethical decisions, and in general tried to make the best decision within the time limits available. They did their best to assess capacity and take proper consent, respect patient autonomy and their duty of care, repeat consultations they felt necessary in a more respectful style, offer reassurance about the purpose of an examination that was carried out without consent or explanation, and manage a complaint and a self-discharge appropriately. Medical ethics was not at the forefront of their reasoning when they were dealing with resource-allocation issues and they were unsure how to deal with problems involving colleagues, but no doubt the weekend shift tomorrow will provide more exposure to these issues and opportunities to approach them differently.

\section{CONCLUSION}

The everyday ethical encounters faced by junior doctors are generally not as dramatic as the big issues discussed at medical school, although the underlying principles, such as consent, confidentiality and resource allocation, remain the same. However, ethical issues come up far more frequently than most junior doctors anticipated as medical students, with many opportunities in an average, routine day, to apply principles and knowledge of medical ethics.

Most medical schools include medical ethics teaching in their course, and most of the junior doctors I have asked about the issues raised in this article found the teaching interesting. Several mentioned that despite opportunities for discussion after the lectures they had not had the clinical experience at the time to know what sort of issues they would encounter. Following an extensive literature review of research involving junior doctors, which demonstrated the range of 'everyday' ethical challenges these doctors face, McDougall and Sokol suggest that it is important for medical ethics teaching to ensure that these typical scenarios are discussed at the medical school stage, even if they seem mundane compared with the more dramatic issues in the field of medical ethics. ${ }^{3}$

Perhaps one of the most difficult challenges for junior doctors is the issue of how to intervene when they see a more senior colleague behave in an ethically unacceptable way, particularly if this colleague is encouraging them to behave in the same way. Doyal describes how medical students at one London medical school helped produce a policy underlining the rights of patients asked to participate in educational activities and subsequently found it much easier to question and decline to participate in unethical behaviour once there was a published policy outlining ethically acceptable standards. ${ }^{4}$ Identifying ethical problems is the first step towards resolving them, but now that we are aware of the problems, we are left with many questions about their resolution. The valuable but daunting task of finding practical solutions to the range of ethical issues encountered on the hospital wards is beyond the scope of this article, but my hope is that this paper will encourage some readers to look for solutions that could be implemented in their own work places.

Competing interests None.

Provenance and peer review Commissioned; externally peer reviewed.

\section{REFERENCES}

1. GMC Education. Medical Students: Professional values and fitness to practice. General Medical Council Guidelines:2008.

2. GMC. Good Medical Practice 2006: Making and Using Visual and Audio Recordings of Patients. General Medical Council Guidelines:2002.

3. McDougall R, Sokol DK. The ethical junior: a typology of ethical problems faced by house officers. J Royal Soc Med 2008;101:67-70.

4. Doyal L. Closing the gap between professional teaching and practice. Br Med J 2001;322:685-6. 